\title{
Téoros
}

Revue de recherche en tourisme

\section{Pour une politique de signalisation du produit touristique}

\section{Danielle Lavoie}

Volume 6, numéro 1, mars 1987

Tourisme et transports

URI : https://id.erudit.org/iderudit/1080525ar

DOI : https://doi.org/10.7202/1080525ar

Aller au sommaire du numéro

Éditeur(s)

Université du Québec à Montréal

ISSN

0712-8657 (imprimé)

1923-2705 (numérique)

Découvrir la revue

Citer cet article

Lavoie, D. (1987). Pour une politique de signalisation du produit touristique. Téoros, 6(1), 33-42. https://doi.org/10.7202/1080525ar d'utilisation que vous pouvez consulter en ligne.

https://apropos.erudit.org/fr/usagers/politique-dutilisation/ 


\section{Pour une politique de signalisation du produit touristique}

par Danielle Lavoie*

Pour le touriste qui circule au Québec, cháque intersection, chaque carrefour peut devenir un véritable cauchemar. Pour retrouver l'hôtel qu'il recherche, le centre de ski qu'il a choisi, l'equipement touristique mentionne dans son guide, il est souvent sans recours.

Par contre, il a pu prendre connaissance, sur des remorques stationnées le long des autoroutes, de tous les debosseleurs du coin.

Pour ces raisons, depuis de nombreuses années, de multiples propriétaires d'équipements touristiques essaient d'amener le ministère des Transports à modifier la signalisation touristique sur les routes du Québec:

Et le ministère des Transports du Québec est prisonnier du problème suivant: décider quel equipement sera signale, à quels endroits sur les routes, comment se fera cette signalisation et qui en défraiera les coûts.

Par ailleurs, des que les divers intervenants dans ce dossier essaient d'arrêter ensemble des solutions sur chacun de ces sujets, les difficultés du départ, loin d'être diminuées, augmentent.

Voyons done dans un premier temps, quels sont les divers intervenants impliqués dans le dossier et le problème que cause leur approche différente.

\section{Les intervenants et la signalisation touristique Le ministère des Transports \\ La sécurité routière}

En vertu du Code de la route, le ministère des Transports détient seul le pouvoir d'implanter la signalisation sur les routes provinciales. Par tradition, le ministère des Transports a concentre ses efforts sur la signalisation de dangers, de prescriptions (ex.: indication des vitesses permises) et d'indications (ex.: nom des municipalites).

Le ministère des Transports a donc édicté les normes de signalisation en tenant

"Chargée de projet au ministère du Tourisme du Quebec

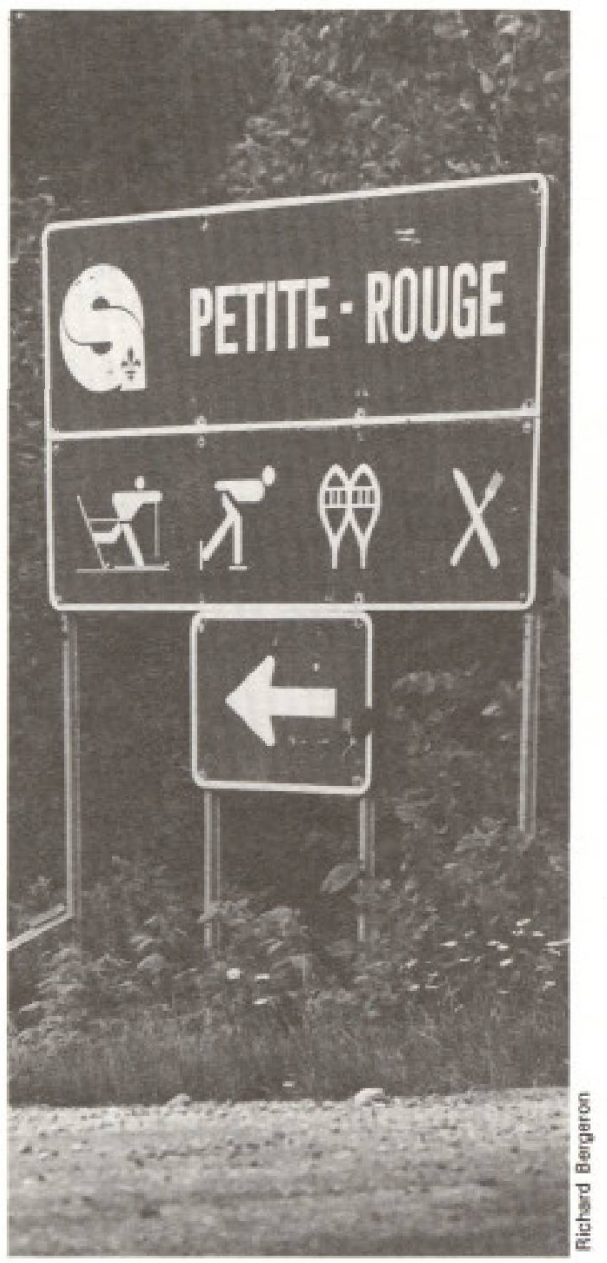

compte des prineipes suivants: l'automobiliste empruntant le réseau routier québécois, entretenu ou non par le Ministere, est assuré de trouver une signalisation homogène, au niveau de la forme, de la couleur et du contenu du message et ce, pour la signalisation de dangers, de prescriptions et d'indications.

\section{La signalisation actuelle}

du produit touristique

Le ministère des Transports, suite aux demandes répétées des ministères (Tourisme, Loisirs, Chasse et Pêche, Affaires culturelles), associations et fédérations (hôteliers, camping, caravaning, centres de ski alpin), décidait à la fin des années 70 de signaler certains équipements et servi- ces liés à l'activité touristique. Cette signalisation touche encore aujourd'hui dix services (ex.: aéroports, haltes routières, bureaux d'informations touristiques), trois attraits (ex.; musées, belvédères, zoos) et deux activités (ex.: golf, ski alpin). Les principales contraintes de cette signalisation résident dans le fait que pour être signale par le minitère des Transports, un service, un attrait ou une activité doit être situé sur une route entretenue par le ministère et âtre identifié uniquement par un pictogramme. Dès qu'il est à l'écart d'une telle route, l'attrait ou l'activité n"existe plus pour le touriste.

De plus, l'équipement touristique doit satisfaire à des criteres de sélection specifiques (ex.: un camping pour être signalé doit offrir 100 emplacements) ce qui ccarte nombre d'équipements utiles au touriste.

On constate donc que les critères actuels ne donnent accès à la signalisation routière qu'à une faible portion du produit recherché par la clientèle touristique.

\section{L'affichage le long des routes}

De plus, il est du ressort du ministère des Transports de definir l'utilisation des abords de toutes les routes du Québec à l'exception de celles des territoires régis par la Loi des cites et villes. La Loi sur les panneaux-réclame et affiches relève donc de son autorite.

Les enseignes ou affiches installées en vertu de cette loi ne présentent pas de principe d'uniformité. Les contrôles se limitent à la dimension et à la localisation de l'enseigne. Comme une bonne partie de l'information destinée au touriste, en termes de services ou d'attraits, est signalée par le panneauréclame, et que n'importe qui peut l'utiliser à sa guise et inscrire le message de son choix, l'image du produit est diminuée sans compter l'effet visuel negatif sur l'environnement.

\section{Les municipalitês}

Les municipalités qui effectuent l'entretien de leur réseau routier sont responsables de la signalisation routière. En ce sens, elles peuvent indiquer le produit touristique uniquement sur le réseau routier municipal. 
Le problème qui se pose alors, c'est que souvent le touriste circule sur les routes provinciales. Or, sur ces routes, la majorité du produit touristique est exclue de la signalisation du ministère des Transports.

Et lorsque les intervenants ont recours à l'affichage à l'extérieur de l'emprise de la route, ils dépendent du bon vouloir des différents propriétaires de terrain et se voient confrontés au même problème à toutes les intersections.

\section{L'industrie touristique}

Les proprietaires d'equipements touristiques recherchent la signalisation pour faire connaître leur produit à la clientèle touristique et rentabiliser leur entreprise. Ils sont confrontés aux limites exposées plus haut. Ils sont pratiquement dans l'impossibilité d'annoncer de façon convenable leur produit et se voient souvent dans l'obligation d'utiliser des palliatifs moins convenables (ex.: utilisation d $\mathrm{d}^{+}$enseignes démesurées, panneaux-réclame ).

En somme, ils reprochent à la réglementation actuelle:

- de fixer des normes à l'échelle du Québec sans tenir compte des particularités régionales: à Rouyn, il faut autant de sites de camping qu'à Québec pour avoir droit à la signalisation?

- de ne pouvoir attirer l'attention du touriste à partir des grands axes routiers et de les diriger jusqu'ù leur destination. ex.: le centre de ski du Mont Sutton n'est pas indiqué sur 1 tautoroute 10 ;

- d'éliminer de la signalisation des équipements majeurs parce qu"ils ne sont pas retenus dans la programmation du ministère des Transports; (ex.: Aquaparc) ou encore des zones touristiques parce qu'elles sont situées en territoires municipaux. (Ex.: Vieux-Quebec Vieux Montréal.)

\section{Le touriste}

Selon les dernières statistiques disponibles, la clientèle touristique a effectué, en 1982 au Québec, plus de 18627000 voyagespersonnes. Plus de $80 \%$ de cette clientele utilise l'automobile lors de ses déplacements. L'activité touristique génère donc de nombreux déplacements routiers.

Le principal besoin du touriste, à part ceux de première nécessité (se loger, se nourrir, s'informer) est de se situer: en effet, à partir de l'information touristique consultée, il désire retourver sur son itinérairé les attraits et activités retenus pour fins de visite et les principaux services mis à sa disposition.

La pratique actuelle aide partiellement le touriste, puisqu'une partie du produit touristique seulement lui est indiquée. Un touriste qui circule sur l'autoroute 20 entre Québec et Montréal ne rencontre aucune signalisation particulière lui indiquant les régions qu'il traverse ni le produit qui lui est offert. Selon la signalisation existante, ces régions n'offrent qu'un ou deux golfs, quelques terrains de camping et stations d'essence.

D'une part, le touriste ne peut retrouver sur sa toute les ́quipements indiqués dans ses guides et d'autre part, il ne peut découvrir les attraits ou activités qui lui sont offerts directement par les divers intervenants et qui pourraient prolonger son séjour au Québec.

Enfin, la majorité des équipements signalés se trouvent sur les axes secondaires ou les routes municipales. Et ces routes ne sont pas les premières empruntes par le touriste.

Le touriste qui doit conduire sa voiture, chercher sal route, découvrir de nouveaux attraits et retrouver ceux qu'il cherche, fait face à une tâche complexe.

Or, la nature et le nombre d'activités auxquelles participeront les touristes, les services qu'ils utiliseront, la possibilité qu'ils fassent un autre voyage au Québec et la promotion qu'ils feront du Québec dans leur milieu sont étroitement liés à la qualité de l'accueil et de l'information qu'ils auront reçus sur le territoire québécois. La signalisation touristique est une composante essentielle de l'accueil. Elle permet de faciliter l'accès au produit touristique, elle reflète l'hospitalitế quếbécoise et assure la promotion du produit touristique aupres des usagers du réseau routier.

C'est en ce sens que le ministere du Tourisme a travaillé en collaboration avec le ministère des Transports pour développer une politique de signalisation du produit touristique québếcois.

\section{Une politique de signalisation touristique}

Pour répondre aux attentes des touristes et des intervenants touristiques, les ministeres du Tourisme et des. Transports ont êlaboré une politique de signalisation qui vise la mise en valeur du produit touristique et son accessibilite pour le touristes.

Cette politique de signalisation touristique fait suite au depot en 1983 par la Direction générale du tourisme d ${ }^{+} u n$ document intitulé "'Vers une nouvelle signalisation routière du produit touristique québécois" ". (1) Ce document dressait l'état de la situation en matière de signalisation touristique et présentait des propositions visant â corriger les problemes identifiés.

En 1984, un comité de travail formé de représentants des ministères des Transports et du Tourisme recevait le mandat de définir une politique de signalisation touristique. En 1986, une proposition de politiqué de signalisation touristique était presenté et accepté par le Conseil des ministres.
Cette politique fait maintenant l'objet d'une consultation aupres des intervenants concernés (municipalités, intervenants touristiques); elle serạ expérimentée à l'été 1987 dans la région touristique du Coeur-duQuébec et implantée sur l'ensemble du réseau routier à l'été 1988. Elle se retrouve dans le document intitulé " $L$ a signalisation du produit touristique québécois ${ }^{* t(2)}$.

Les principes sur lesquels repose la politique de signalisation touristique sont:

- L'accessibilité de la signalisation à tout equipement reconnu touristique;

- L'acheminement complet de cette signalisation jusqu'au site de l'équipement sur la totalité du réseau routier québécois même si elle doit emprunter des routes de compétence municipale;

- L'uniformité de la signalisation quant a la dimension des panneaux, leur localisation, leur couleur et leur contenu (un pictogramme, le nom de l'equipement, la distance et la direction);

- L'autofinancement du système: le proprićtaire d'un équipement touristique éligible à la signalisation défraie les coûts reliés à la signalisation de son equipement.

Cette nouvelle politique s'appliquera a l'interieur de l'emprise des routes et utilisera deux types de panneaux:

La signalisation sur panneaux bruns Cette signalisation existe présentement sur les routes. Elle est modifiée par l'entrée en vigueur de la politique de signalisation touristique. Dorénavant, elle comprend les sites et installations touristiques qui $n^{7}$ ont pas de promoteur, comme les ressources naturelles et ceux dont la propriété et l'exploitation relèvent de l'État comme les pares provinciaux ou nationaux.

\section{La signalisation sur panneaux bleus} Elle comprend les equipements touristiques de nature commerciale, dont la propritété et l'exploitation relèvent du secteur privé. Les couts de cette signalisation sont assumés par le propriétaire de l'équipement et elle exige la signature de contrats entre les requérants et le ministère des Transports.

La mise en place de cette politique de signalisation dans l'emprise des routes ne saurait toutefois solutionner tous les problèmes constatés. Aussi, les ministères impliqués dans ce dossier comptent intervenir à l'extérieur de l'emprise des routes et dans les haltes routières.

À l'extérieur de l'emprise des routes: L'affichage est réglementé par la Loi sur les panneaux-reclame et affiches. Cette dernière fait actuellement l'objet d'une révision au ministère des Transports. 
WHEATCROFT, Stephen, "The changing economics of international air transport ", Tourism Management, vol. 3, no 2, (June 1982), pp. 71-82.

\section{Transport maritime}

HANBY, Betsy, "1985 outlook for cruise lines'", in 1985 outlook for travel and tourism: proceedings of the US Travel Data Center - 1985 Travel outlook forum, Washington, D.C., USTDC, 1985, pp. 115-119.

CANADA, Bureau des conseillers en gestion, The tourism industry and the role of ferry services, Ottawa, BCG, 1976, $71 \mathrm{p}$.

KANE, Arthur W, and Jean-Claude POTIER, "The cruise catalysts", in Travel and Tourism Research Association Fifteenth Annual Conference, Salt-Lake City, TTRA, 1984, pp. 171-181.

LEWIS, Jay and Dan SAREL, "Cruise industry in the USA: matching supply and demand by 1990 " Travel and Tourism Analyst, (August 1986), pp. 43-55.

LUCAS GIRARDVILLE, Jean-Marie, Les croisières au Québec, Québec, Ministère du Tourisme, $1980,243 \mathrm{p}$.
MARTI, Bruce E.., "Cruising: smallvessel population characteristics" "Journal of Travel Research, vol. XXIV, no 4 (Spring 1986), pp. 25-28.

\section{Automobile et tourisme}

ASPECT ASSOCIATES, U.S. automobile travel to Canada 1972-1983: an econometric evaluation, Toronto, Aspect Associates for Tourism Industry Association of Canada, 1984,56 p.

BREDEMEIR, Judi, "The US car rental industry: surviving the 1980's'". Travel and Tourism Analyst (March 1986), pp. 47-55.

BRYAN, William, "Improved mileage discretionary income and iravel for pleasure", Journal of Travel Research, vol. XX, no 1 (Summer 1981), pp. 28-29.

CORSI, Thomas M. and Milton E. HAWEY, "Changes in Vacation Travel in Response to Motor fuel Shortages and higers prices", Journal of Travel Research, vol. XVII, no 4 (Spring 1979), pp. 7-11.

DIONNE, France, Rapport du comité Dionne sur les haltes routières, Québec, 1986.
KAMP, B. Dan, John L. CROMPTON and David M. HENSARLING, "The reactions of travelers to gasoline rationing and to increases in gasoline prices", Journal of Travel Research, vol. XVIII, no 1 (Summer 1979), pp. 37-41.

QUÉBEC (Province), Ministère des Transports et Ministère du tourisme, Projet de politique de signalisation du produit touristique, Québec, 1984, 62 p.

TRENT, Roger B. and Cecil POLLARD, "Gasoline prices and Discretionary auto travel: evidence from a panel survey", Journal of Travel Research, vol. XXI, no 4 (Spring 1984), pp. 12-16.

WILBUR, Allan, "Oulook for awto vacation market segments", in 1985-86 outlook for travel \& tourism: proceedings of the eleventh annual travel outlook forum, Washington, D.C., US Travel Data CEnter, 1986, pp. 112-124, f
La demande de transport

(Suite de la page 29)

(4) JAKLE, A. The tourist, travel in twentiethcentury North-America. University of Nebraska Press, Lincoln, 1985.

(5) WILSON, A., "A Statistical Theory of Spatial Distribution Models". Transportation Research. vol. I, N.Y, 1967 .

(6) OUANDT, R. The Demand for Travel: Theory and Measurement, Heath Lexington Books, Lexington, Mass., 1970.

(7) GOUDWIN, P., La valeur du temps, C.E.M.T. Paris, 1976.

(8) COOLEY, T,F, Forecasting the demand for travel, U.S. Department of Transportation, Washington, 1979.

(9) STOPHER, R.، MEYBURG, A., Behavioral Travel Demand Models, Lexington Books, Toronto, 1976.

(10)BROG, $W$, ROGERS, $K$, New Horizons in Travel-Behavioral Research, Lexington Books, Toronto, 1981

(11) BRAUDEL, F., "La longue dusée", in Eerits sur I'histoire, Flammarion, Paris, 1969 , p. 50.

I12IBAAUDEL, F., op. cit., p. 46 .

(13) NAHON, G. SALOMON, M., L'élaboration des prévisions de marché, Dunod, Paris, 1977, p. 21.

[14/Voyages entre le Canada et les autres pays, Statistique Canada, Cataloques (66-001).

(15) II s'agit ici du taux doaccroissement annuel moyen (TAAM) calculé avec la movenne géométrique.

(16)Les cing formes d'ajustement ont été calculées pour les quatre modes de transport, aै l'aide du progiciel S.A.S. sur l'ordinateur. Amdahl de l'Université du Québec à Montréal.
(17)BOER.JAN, P NANHOVE, N., "The Tourism Demand Reconsidered in the Context of the Eca nomic Crisis", in Revue de Tourisme, vol. 39, no 2. Berne, 1994

I18ISTUBBS, P., TYSON, W., DALVI, M., Transport Economics, George Allen Umwin, London, 1980, pp. $12-19$

I19ICHRISTIE, A.., MORRISON, A., The Tourism System, Prentice Hall, N.J., 1985, pp. 169-1970.

(20) LANG, R., Train, avrion ou automobile! Les choix de l'usager, Economica, Paris, 1990, p. 131

(21)BONSOR, N. Transportation Economics, Butterworths, Toronto, 1984 , pp. 56-58.

(22) VIARD, J., Penser les vacances, Actes-Sud, Marseille, 1984, p. 127
Pour une politique...

(Suite de la page 34)

\section{Dans les haltes routières}

Des bureaux d'informations touristiques sont déja localisés dans certaines haltes routières du ministère des Transports. Le ministère du Tourisme compte implanter, en collaboration avec les Associations touristiques régionales, des relais d'information touristique dans des haltes routières situés à des endroits stratégiques. Les relais d'information touristique sont en fait des panneaux informatifs et promotionnels. Ils visent par la transmission d'informations à assurer un sentiment de suivi entre le touriste et le réseau de bureaux d'informations touristiques. Ils s'intègrent également à la signalisation touristique, car ils ont aussi comme objectif d'aider à acheminer la clientèle touristique jusqu'à l'attrait, l'activité ou le service choisi.

D'ores et déjà, plus de gens trouveront satisfaction face à la signalisation touristique. Nous croyons que les démarches entreprises contribueront à rendre le Québec touristique plus accueillant. $f$

(1) LAVOIE Danielle, Vers une nouvelle signalisation routière du produit touristique québécois. Ministère de I'lndustrie, du Commerce et du Tourisme, 1983, 32 pagess.

(2) Ministère des Transports, Ministëre du Tourisme La signalisation du produit touriatique quobbcois. 1987, 15 pages. 